\title{
Cos Transcranial magnetic resonance imaging-guided focused ultrasound thalamotomy for tremor: technical note
}

\author{
Tony R. Wang, MD, ${ }^{1}$ Aaron E. Bond, MD, PhD, ${ }^{1}$ Robert F. Dallapiazza, MD, PhD, ${ }^{2}$ \\ Aaron Blanke, MS, ${ }^{3}$ David Tilden, BS, ${ }^{3}$ Thomas E. Huerta, BS, RT, ${ }^{4}$ Shayan Moosa, MD, ${ }^{1}$ \\ Francesco U. Prada, MD, ${ }^{1,5}$ and W. Jeffrey Elias, MD ${ }^{1}$
}

\begin{abstract}
'Department of Neurological Surgery, and ${ }^{4}$ Department of Radiology, Neuroradiology Division, University of Virginia Health System, Charlottesville, Virginia; ${ }^{2}$ Division of Neurosurgery, Toronto Western Hospital University Health Network, Toronto, Ontario, Canada; ${ }^{3}$ Insightec Ltd., Dallas, Texas; and ${ }^{5}$ Department of Neurosurgery, Fondazione IRCCS Istituto Neurologico C. Besta, Milan, Italy
\end{abstract}

\begin{abstract}
Although the use of focused ultrasound (FUS) in neurosurgery dates to the 1950s, its clinical utility was limited by the need for a craniotomy to create an acoustic window. Recent technological advances have enabled efficient transcranial delivery of US. Moreover, US is now coupled with MRI to ensure precise energy delivery and monitoring. Thus, MRIguided transcranial FUS lesioning is now being investigated for myriad neurological and psychiatric disorders. Among the first transcranial FUS treatments is thalamotomy for the treatment of various tremors. The authors provide a technical overview of FUS thalamotomy for tremor as well as important lessons learned during their experience with this emerging technology.
\end{abstract}

https://thejns.org/doi/abs/10.3171/2017.10.FOCUS17609

KEY WORDS focused ultrasound; FUS; tremor; technique

$\mathrm{H}$ IGH-INTENSITY focused ultrasound (HIFU) dates to the 1950s, when it was used to create lesions in the feline brain without damage to the intervening parenchyma. ${ }^{10,11}$ Following this, HIFU was used effectively for movement disorders, neuropsychiatric conditions, and glioblastoma. 13,19,21,24 Despite these early studies, widespread adoption of HIFU was limited by the need for a craniotomy, because most US energy is either absorbed or reflected by the skull. ${ }^{27}$

Recent advances in transducer design ${ }^{5,6,14,15}$ and the development of wave phase correction algorithms to adjust for US distortion ${ }^{3,4}$ by the skull have obviated the need for craniotomy and allowed for transcranial HIFU. Advances in MRI and MR thermography have additionally allowed for accurate targeting ${ }^{23}$ and temperature monitoring ${ }^{17}$ during sonication. These developments have subsequently reinvigorated interest in HIFU for neurological conditions. Of these conditions, we aimed our early interest in transcranial HIFU to essential tremor (ET). We thought that the sensitive symptom of tremor, which is easily assessed during the procedure, would respond to subthreshold sonications for optimal localization prior to ablation. ${ }^{8}$ Three uncontrolled trials investigating unilateral, transcranial MRI-guided FUS thalamotomy for ET showed promising results. ${ }^{1,8,20} \mathrm{~A}$ subsequent multicenter randomized controlled trial ${ }^{9}$ confirmed the results and led to FDA approval of unilateral FUS thalamotomy for medication-refractory ET (Table 1). In this report, we outline the technique of this procedure and important lessons learned during our experience with this technology.

\section{Technique \\ Preoperative Imaging}

Prior to FUS, volumetric MRI and noncontrast CT of the brain are obtained. From the CT, the skull density ratio (SDR) is calculated. The SDR represents the global average of the ratio in Hounsfield units of the cancellous to cor-

ABBREVIATIONS AC-PC = anterior commissure-posterior commissure; ET = essential tremor; FUS = focused ultrasound; HIFU = high-intensity FUS; SDR = skull density ratio; Vim = ventral intermediate.

SUBMITTED September 29, 2017. ACCEPTED October 30, 2017.

INCLUDE WHEN CITING DOI: 10.3171/2017.10.FOCUS17609. 
TABLE 1. Sonication parameters for FUS trials for treatment of ET

\begin{tabular}{lcccccc}
\hline \multicolumn{1}{c}{ Authors \& Year } & $\begin{array}{c}\text { No. } \\
\text { of Pts } \\
\text { Treated }\end{array}$ & $\begin{array}{c}\text { Power } \\
(\mathrm{W})\end{array}$ & $\begin{array}{c}\text { Sonication } \\
\text { Duration } \\
(\mathrm{sec})\end{array}$ & Mean Final Sonication Energy $(\mathrm{J})$ & $\begin{array}{c}\text { Mean No. of } \\
\text { Sonications }\end{array}$ & Mean Peak Temp $\left({ }^{\circ} \mathrm{C}\right)$ \\
\hline Lipsman et al., 2013 & 4 & $300-1250$ & $10-25$ & NA & $22.5 \pm 7.6$; range $12-29$ & $59.3 \pm 2.9 ;$ range 56-63 \\
\hline Elias et al., 2013 & 15 & NA & $10-20$ & $10,320 \pm 4,537$; range 6,500-20,800 & $17.9 \pm 4.6$; range 11-26 & $58.5 \pm 2.5 ;$ range 54-63 \\
\hline Chang et al., 2015 & 11 & NA & $10-20$ & 24,000 max & NA & $53 \pm 3.3 ;$ range 48-61* \\
\hline Elias et al., 2016† & 56 & NA & NA & $14,497 \pm 6,696 ;$ range 3,500-34,860 & $18.5 \pm 5.2$ & $55.6 \pm 2.3 ;$ range 50-60.7 \\
\hline
\end{tabular}

$\mathrm{NA}=$ not applicable; $\mathrm{pts}=$ patients; temp = temperature.

* Includes 3 patients with peak temperatures $<50^{\circ} \mathrm{C}$; in these patients treatment was deemed a failure.

$\dagger$ Randomized, sham-controlled trial; all others are open-label trials.

tical bone of the skull. ${ }^{2}$ The SDR has a theoretical range of $0-1$, with lower values indicative of a large discrepancy between the Hounsfield units of the cancellous to cortical bone; SDR values approaching 1 indicate less of a discrepancy. In our experience, SDR values $>0.4$ are amenable to more efficient FUS lesioning. The CT study additionally allows identification of the frontal sinuses and intracranial calcifications (e.g., choroid plexus, pineal gland, and falx). These structures can impede US transmission and are marked as no-pass regions on the planning software, areas through which no acoustic energy is delivered.

\section{Preoperative Considerations and Placement of Stereotactic Frame}

Intravenous access is obtained in the patient's nontreated hand, and compression stockings are placed. The patient's hair is clipped and carefully shaved to avoid any trapped microbubbles that could cavitate and cause scalp burns. A stereotactic frame that is modified for compatibility with the FUS transducer and MRI environment is used. The frame is affixed low on the head (near the orbital rim) in the approximate orientation of the anterior commissure-posterior commissure (AC-PC) (Fig. 1).

\section{Anesthetic Considerations}

The stereotactic frame is placed with local anesthesia (10:10:1 ratio of $1 \%$ lidocaine/ $0.5 \%$ bupivacaine/ $8.4 \%$ sodium bicarbonate). After frame placement, intravenous acetaminophen $(1 \mathrm{~g})$ and intravenous lorazepam (0.25-0.5 $\mathrm{mg}$ ) are administered for comfort during the procedure. This dose of anxiolytic agent increases patient comfort, with minimal effect on tremor during testing that occurs later in the procedure. For patients with continued anxiety and/or scalp pain during sonication, additional lorazepam $(0.5-1.0 \mathrm{mg})$ and/or fentanyl $(25-50 \mu \mathrm{g})$ can be given. Caution should be exercised when giving anxiolytics and/ or analgesics because both may suppress tremor, impairing physiological confirmation of the thalamic target while also confounding the treatment effect.

\section{Patient Positioning}

A silicone membrane is placed over the scalp as low as possible and the patient is placed supine on the MRI table. The frame is secured to the MRI cradle and the silicone membrane is attached to the US transducer (NeuroAblate 4000; Insightec). Between the dome-shaped US transduc- er and the silicone membrane is a space that is eventually filled with chilled, degassed water, which serves as a coupling medium for US transmission and a cooling agent for the scalp. The US transducer is finally grossly aligned in the $\mathrm{X}, \mathrm{Y}$, and $\mathrm{Z}$ planes to the thalamic target and tilted $5^{\circ}-10^{\circ}$ in the dorsal direction to approximate the AC-PC plane.

\section{Procedural Imaging and Stereotactic Planning of Thalamic Target}

Localizer MRI (T2-weighted) images are obtained so that sagittal T2-weighted images can be prescribed parallel to midline. From the most midline slice, the AC and $\mathrm{PC}$ are identified. Reference T2-weighted images are then obtained in the axial and coronal planes defined by the $\mathrm{AC}$ and PC and orthogonal to the midline. The thalamic target is now planned on the axial slice demonstrating both AC and $\mathrm{PC}$ by using indirect targeting. The ventral intermedi-

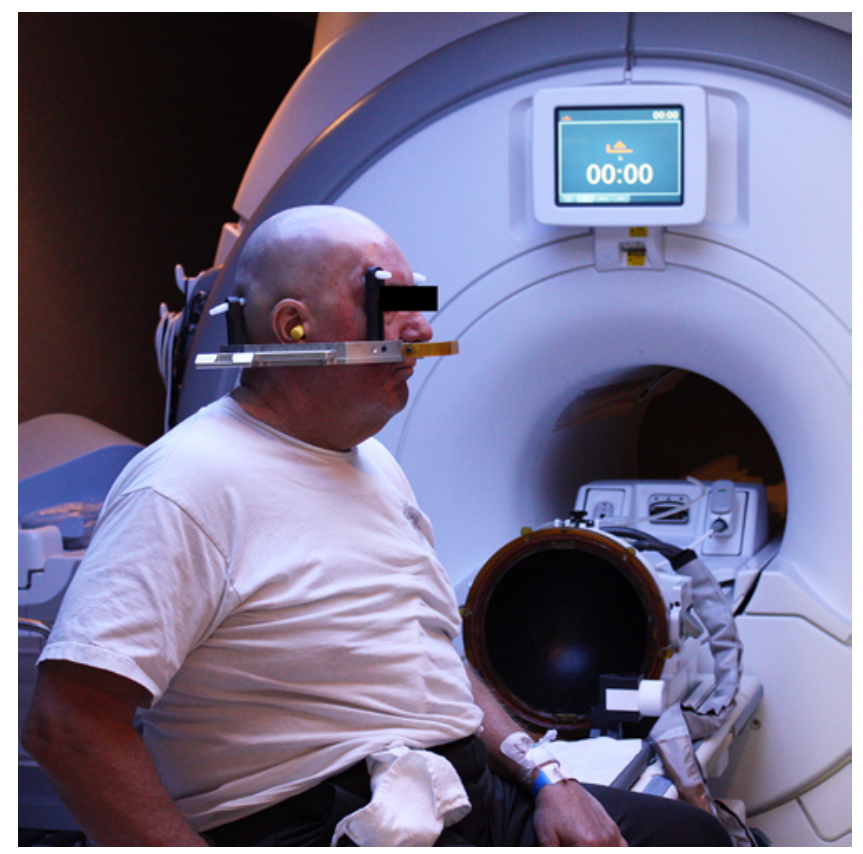

FIG. 1. Patient seated on the MRI table prior to left-sided FUS thalamotomy for ET. The stereotactic frame is placed low and grossly parallel to the AC-PC plane (lateral canthus to external auditory canal) to ensure maximal exposure of the cranium to the US transducer. 
ate (Vim) nucleus of the thalamus is estimated at 14-14.5 $\mathrm{mm}$ lateral to the midline, or $11 \mathrm{~mm}$ lateral to the third ventricle wall in cases of ventriculomegaly. The anteriorposterior dimension is one-fourth the AC-PC length behind the midcommissural point or $6 \mathrm{~mm}$ anterior to the PC (Fig. 2). Successful targeting has also been achieved with MR tractography. ${ }^{28}$

The preoperative volumetric CT is next fused with the MR image, which ensures that the proper skull correction algorithms are applied to allow for efficient transcranial sonication. Fiducial markers are then placed on the axial, coronal, and sagittal reference images, which serve as a baseline for movement detection scans that occur every third sonication. If movement $>2 \mathrm{~mm}$ around any fiducial marker in MRI space is detected, the sonication is halted and the operator is notified of potential movement. Additional no-pass regions are now delineated. These nopass regions represent air-filled folds of the silicone membrane created near the scalp once the space between the US transducer and the silicone membrane is filled with chilled, degassed water. For every no-pass region marked, additional elements - from a maximum of 1024-within the transducer are shut off to prevent US transmission. Having more elements during an FUS procedure portends a higher likelihood of attaining ablative temperatures; 700 elements would be the lower limit of a successful procedure (Fig. 2). Last, the US transducer is precisely moved so that its natural focus matches the planned target.

\section{Treatment Phase}

The treatment phase consists of 3 stages: ensuring alignment of the US focus, confirming the thalamic target, and therapeutic sonication.

\section{First Stage}

During alignment, low-energy sonications (typically $1500-3000 \mathrm{~J}$ ) are performed to create a thermal spot that can be localized with single-slice, 2D MR thermography. Thermography scans are acquired approximately every 3 seconds during sonication, and provide the operator with 2 separate temperatures: the maximum and average temperatures of a 9-voxel-sized box. The MR thermography is precise to $1^{\circ} \mathrm{C}-2^{\circ} \mathrm{C}$, and assumes a baseline body temperature of $37^{\circ} \mathrm{C}$; MR thermography does not measure absolute temperature. A thermal spot with a peak or average temperature between $40^{\circ} \mathrm{C}$ and $45^{\circ} \mathrm{C}$ can be generated to align the US focus. These temperatures are unlikely to damage tissue or cause neurological effects because they are below thermal thresholds for tissue damage in the brain. ${ }^{12,16,18}$

The accuracy of thermal rise is only assessed in the phase orthogonal to the encoding direction due to a known thermal shift in the frequency-encoding direction of MR thermography. ${ }^{26}$ For example, during a sonication in which an axial MR thermography scan is obtained in the anterior-posterior frequency direction, accuracy in the medial-lateral phase direction is assessed. If the area of maximal temperature rise correlates with the target in the medial-lateral direction, the medial-lateral accuracy is confirmed and we proceed to check the remaining orientations (anterior-posterior and superior-inferior). If the area of maximal temperature rise is slightly off in the medial-
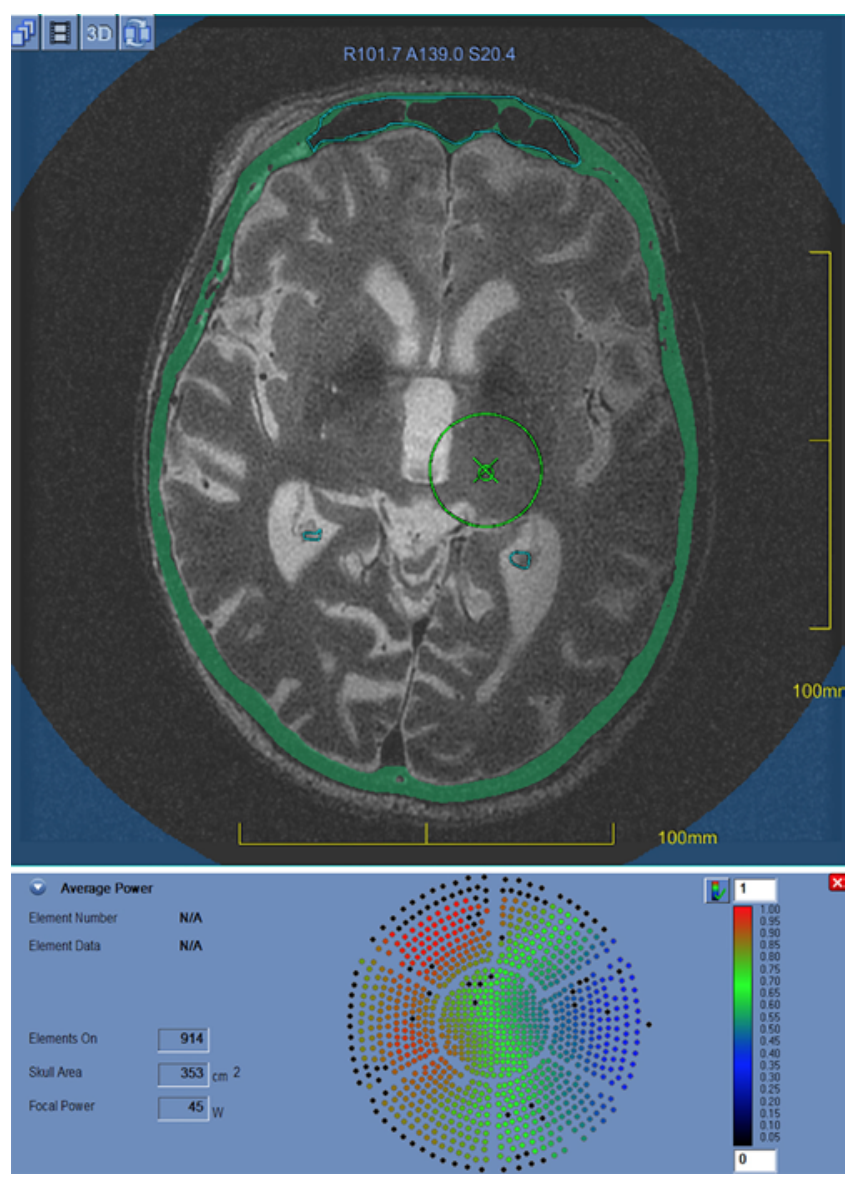

FIG. 2. Upper: Initial planned thalamic target. The $X$ denotes our planned target and the small circle denotes the natural focus of the US transducer. Also pictured is the CT overlay with no-pass regions marking the frontal sinus and calcifications of the choroid plexus, denoted by the blue outline. Lower: Configuration of US elements following marking of all no-pass regions. For every no-pass region marked, US elements are turned off, denoted by black dots. The elements on the right side of the transducer have a higher intensity because acoustic energy must travel farther to reach the planned left thalamic target.

lateral direction, we proceed to check the medial-lateral accuracy in another orientation and/or direction. The MR thermography scan can be obtained in axial, coronal, and sagittal planes. Within each plane, scans can be acquired in 2 possible frequency directions for a total of 6 orientation and/or direction configurations. If the alignment is still off in an additional orientation and/or direction, transducer elements can be electronically steered to correct the location of peak energy deposition and to align with the desired target. Once the accuracy is confirmed in all 3 directions, we proceed to confirmation of the thalamic target.

\section{Second Stage}

When confirming the thalamic target, energy is increased to obtain temperatures in the low $50 \mathrm{~s}\left({ }^{\circ} \mathrm{C}\right)$. Our experience has been that neurological effects or tremor suppression do not occur until this level, and that the effect is due to thermal neuromodulation. From this point forward, following every sonication the patient is assessed 

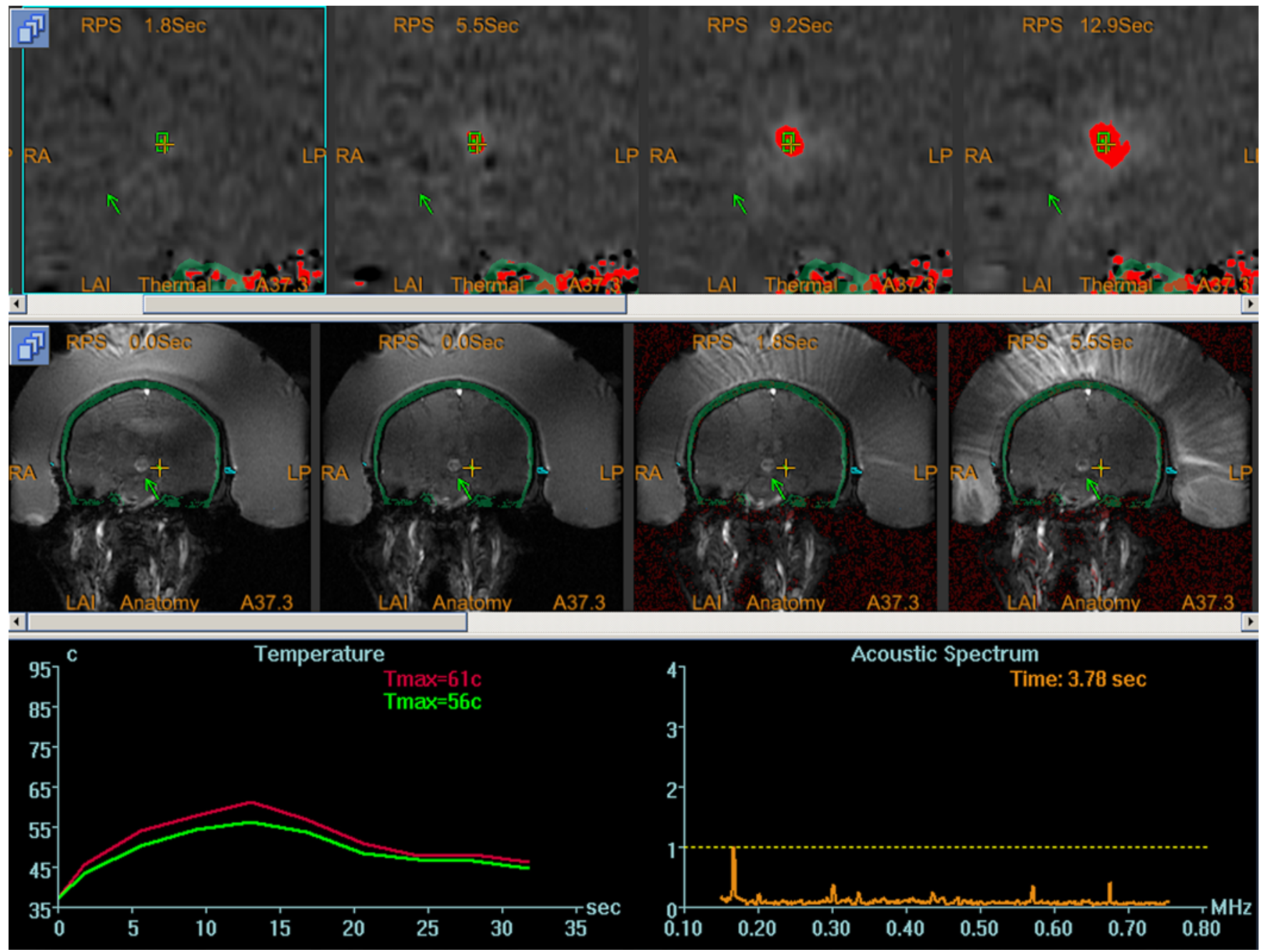

FIG. 3. A therapeutic ablation with the following parameters: power, $648 \mathrm{~W}$; duration, 13 seconds; energy, $8424 \mathrm{~J}$. An MR thermography scan was obtained in the coronal plane; temperature readings are provided approximately every 3 seconds. Peak and average voxel temperatures are $61^{\circ} \mathrm{C}$ and $56^{\circ} \mathrm{C}$, respectively, measured at the 12.9-second thermal trace (upper row, rightmost panel). In the lower right panel, the acoustic spectrum is recorded during each sonication, and from this a potential cavitation can be detected.

for neurological and/or sensory changes and tremor improvement. Sonications producing average temperatures just over $50^{\circ} \mathrm{C}$ can elicit transient paresthesias or sensory changes in the contralateral fingers, hand, lips, and/or tongue. Patients with advanced age, Parkinson's disease, or neuropathy may have less reliable sensory mapping in response to thermal neuromodulation. If somatosensory thalamus can be identified with thermal neuromodulation, the target for ablation (presumed Vim) can be confidently adjusted electronically in the anterior and/or lateral dimensions. If somatosensory mapping does not occur, the target for ablation is redirected to the indirect Vim target.

\section{Third Stage}

For therapeutic ablation, energy is increased to achieve temperatures in the high 50 s to low 60 s $\left({ }^{\circ} \mathrm{C}\right.$; Fig. 3). Our goal is to ablate the Vim at the base of the thalamus (ACPC level) until tremor suppression occurs. After tremor suppression, we review the number of sonications that occurred from $56^{\circ} \mathrm{C}$ to $60^{\circ} \mathrm{C}$ to ensure at least 2 sonications in this therapeutic range. Finally, we enlarge the lesion dorsally by readjusting the target by $2 \mathrm{~mm}$ with additional therapeutic ablation(s). When the procedure seems complete, the water bath is drained to allow for a higherquality T2-weighted MRI. The ablation will be seen as a T2-hyperintense lesion. Thus, the decision to stop the procedure depends mostly on clinical outcome, but also on the thermal dose and intraoperative imaging.

\section{Postoperative Care}

Initially our practice was to admit patients for observation; however, we now perform FUS thalamotomy as an outpatient procedure. No activity restrictions are placed; however, patients are cautioned for possible change of balance in the first few postprocedural days. On the morning following the procedure, volumetric MRI is performed to evaluate the thalamotomy (Fig. 4). 

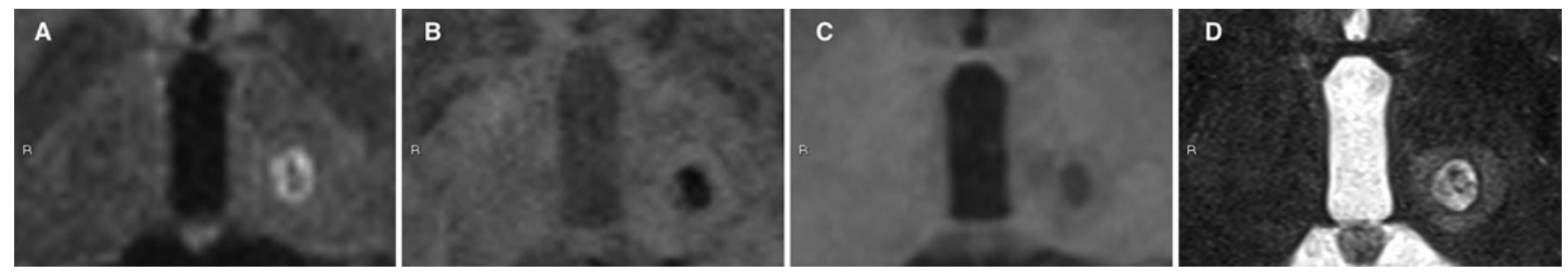

FIG. 4. Left-sided FUS thalamotomy for ET; postprocedure day $1 \mathrm{MRI}$ with diffusion-weighted imaging (A), susceptibility-weighted imaging (B), T1-weighted sequences (C), and T2-weighted sequences (D). Diffusion-weighted changes are the earliest MRI findings with FUS ablations. A hypointense signal on susceptibility-weighted imaging indicates hemoglobin and degraded blood products, even in the absence of overt intracranial bleeding. The findings on T1-weighted imaging may not become apparent until 1 day after the procedure. The perilesional edema seen on T2-weighted imaging peaks at 1 week and resolves by 1 month.

\section{Troubleshooting \\ Intraoperative Head Pain}

Head pain during sonications of increasing energy probably stems from the stimulation of pain-sensitive structures, such as the scalp and periosteum. ${ }^{7}$ Although the pain is transient, some patients may electively prematurely stop sonications because of this pain. Accordingly, we use strategies highlighted in the Anesthetic Considerations section to mitigate head pain during sonication. If head pain persists, the operator can increase sonication duration. This allows the operator to deliver increasing amounts of energy, albeit at a lower intensity, which is often better tolerated by patients.

\section{Abnormal Shape of Thermal Dose}

Because the US transducer is hemispherical in design, the shape of the lesion is slightly elliptical in the superiorinferior orientation. Having heating spread in the inferiorlateral direction risks lesioning of the posterior limb of the internal capsule. Occasional MR thermography scans in the coronal orientation can assess for this spread. Medial-lateral heating can occur in patients with locally high temporal SDR values. The temporal regions are subject to increased efficiency (relative to other areas of the skull) of US transmission, which may lead to unwanted mediallateral heating. Such unwanted heating, which places the posterior limb of the internal capsule at risk, can be mitigated with masking. Masking is a method in which the US elements at the periphery corresponding to the temporal regions are turned off or where energy output is restricted. The US elements around the entire periphery may also be turned off in a technique called apodization, but this feature can reduce the likelihood of achieving an adequate thermal rise.

Even though increasing the sonication time increases energy delivery, it does lead to possible spreading of the thermal dose in multiple planes. Although independently increasing power or sonication time will increase energy, when feasible we advocate increasing power first because this will result in better compactness of the thermal lesion. Problems with spread of the thermal lesion may also be seen in patients with hyperostosis frontalis interna. In such patients, it may be necessary to mark portions of the thickened inner table as a no-pass region. Additionally, the transducer can be tilted past the AC-PC plane such that the most anterior-inferior transducer elements are superior to the thickened regions.

\section{Difficulty Achieving High Temperatures}

The inability to achieve ablative temperatures despite increases in acoustic energy is not yet completely understood; ${ }^{1,2,18}$ however, a recent study found that higher SDR values and smaller skull volumes correlated with a higher likelihood of achieving ablative temperatures. ${ }^{2}$ Consequently, we recommend careful review of the patient's preoperative CT studies to ensure proper patient selection.

Another consideration is the number of US elements, which was found to trend positively $(p=0.06)$ toward higher temperatures. ${ }^{2}$ Several steps can be taken to maximize the number of elements. Low placement of the stereotactic frame and silicone membrane ensures maximal exposure of the patient's head to the US transducer and its elements. The US transducer is also tilted slightly in the dorsal direction, which removes structures in the anteriorinferior direction (i.e., no-pass regions such as the frontal sinuses and silicone membrane folds) from the field of the US transducer. Even no-pass regions outside the field of the transducer will result in elements being shut off due to the far-field effect. ${ }^{29}$ Consequently, no-pass regions outside the transducer overlay should be deselected because this will result in additional US elements.

\section{Occurrence of Cavitation}

The HIFU procedure may induce sustained or inertial cavitation. This occurs when an acoustic wave, moving through tissue, extracts gas that coalesces to form microbubbles. These microbubbles oscillate and can cause uncontrolled damage and hemorrhage..$^{22,25,30}$ The US transducer has cavitation detectors that trigger a sonication halt if cavitation is above a set threshold. The cavitation detection system is limited in that it does not specify where cavitation is occurring. Prophylactic steps to avoid cavitation include the following: close shaving of the head, use of degassed water ( $<2$ ppm of dissolved oxygen), ensuring no leaks from the silicone membrane or visible bubbles, minimizing silicone membrane folds, and proper marking of the silicone membrane no-pass regions.

\section{Patient Movement}

If movement is detected, the operator should examine 
the motion detection images, especially the CT overlay, anatomical landmarks, and the marked fiducials. Often, a single errant fiducial will trigger motion detection. Deleting this fiducial and reacquiring a motion detection scan will frequently solve this problem. If true motion has occurred, multiple fiducials should move. In this case, we recommend reacquiring planning scans and comparing them to the original planning scan. Specific anatomical landmarks are compared in 3D MRI space to determine whether motion occurred. If motion has occurred, the operator will have to restart the procedure by acquiring new planning images and replanning the thalamic target. The operator will have to identify the source of movement to ensure that there is no movement on the subsequent treatment. Steps to avoid patient movement include secure placement of the stereotactic frame, properly fixing the stereotactic frame to the MRI cradle, and locking the transducer in all degrees of freedom. Finally, the patient may be taken out of the MRI bore to facilitate intraprocedural testing; if the patient is not precisely repositioned in the MRI isocenter, motion detection alarms will be triggered.

\section{Conclusions}

Given the recent FDA approval of unilateral FUS thalamotomy for medication-refractory ET, we anticipate increased interest from both patients and physicians. Here we have outlined our preferred technique and troubleshooting tips for transcranial MRI-guided FUS thalamotomy for tremor.

\section{Acknowledgments}

Dr. Prada receives educational support from the Focused Ultrasound Foundation, Charlottesville, VA.

\section{References}

1. Chang WS, Jung HH, Kweon EJ, Zadicario E, Rachmilevitch I, Chang JW: Unilateral magnetic resonance guided focused ultrasound thalamotomy for essential tremor: practices and clinicoradiological outcomes. J Neurol Neurosurg Psychiatry 86:257-264, 2015

2. Chang WS, Jung HH, Zadicario E, Rachmilevitch I, Tlusty T, Vitek S, et al: Factors associated with successful magnetic resonance-guided focused ultrasound treatment: efficiency of acoustic energy delivery through the skull. J Neurosurg 124:411-416, 2016

3. Clement GT, Hynynen K: Correlation of ultrasound phase with physical skull properties. Ultrasound Med Biol 28:617-624, 2002

4. Clement GT, Hynynen K: A non-invasive method for focusing ultrasound through the human skull. Phys Med Biol 47:1219-1236, 2002

5. Clement GT, Sun J, Giesecke T, Hynynen K: A hemisphere array for non-invasive ultrasound brain therapy and surgery. Phys Med Biol 45:3707-3719, 2000

6. Clement GT, White J, Hynynen K: Investigation of a largearea phased array for focused ultrasound surgery through the skull. Phys Med Biol 45:1071-1083, 2000

7. Connor CW, Hynynen K: Patterns of thermal deposition in the skull during transcranial focused ultrasound surgery. IEEE Trans Biomed Eng 51:1693-1706, 2004

8. Elias WJ, Huss D, Voss T, Loomba J, Khaled M, Zadicario E, et al: A pilot study of focused ultrasound thalamotomy for essential tremor. N Engl J Med 369:640-648, 2013
9. Elias WJ, Lipsman N, Ondo WG, Ghanouni P, Kim YG, Lee $\mathrm{W}$, et al: A randomized trial of focused ultrasound thalamotomy for essential tremor. N Engl J Med 375:730-739, 2016

10. Fry WJ, Barnard JW, Fry EJ, Krumins RF, Brennan JF: U1trasonic lesions in the mammalian central nervous system. Science 122:517-518, 1955

11. Fry WJ, Mosberg WH Jr, Barnard JW, Fry FJ: Production of focal destructive lesions in the central nervous system with ultrasound. J Neurosurg 11:471-478, 1954

12. Harnof S, Zibly Z, Cohen Z, Shaw A, Schlaff C, Kassel NF: Cranial nerve threshold for thermal injury induced by MRI-guided high-intensity focused ultrasound (MRgHIFU): preliminary results on an optic nerve model. IEEE Trans Ultrason Ferroelectr Freq Control 60:702-705, 2013

13. Heimburger RF: Ultrasound augmentation of central nervous system tumor therapy. Indiana Med 78:469-476, 1985

14. Hynynen K, Clement GT, McDannold N, Vykhodtseva N, King R, White PJ, et al: 500-element ultrasound phased array system for noninvasive focal surgery of the brain: a preliminary rabbit study with ex vivo human skulls. Magn Reson Med 52:100-107, 2004

15. Hynynen K, McDannold N, Clement G, Jolesz FA, Zadicario E, Killiany R, et al: Pre-clinical testing of a phased array ultrasound system for MRI-guided noninvasive surgery of the brain - a primate study. Eur J Radiol 59:149-156, 2006

16. Hynynen K, Vykhodtseva NI, Chung AH, Sorrentino V, Colucci V, Jolesz FA: Thermal effects of focused ultrasound on the brain: determination with MR imaging. Radiology 204:247-253, 1997

17. Ishihara Y, Calderon A, Watanabe H, Okamoto K, Suzuki Y, Kuroda K, et al: A precise and fast temperature mapping using water proton chemical shift. Magn Reson Med 34:814823,1995

18. Jung HH, Kim SJ, Roh D, Chang JG, Chang WS, Kweon EJ, et al: Bilateral thermal capsulotomy with MR-guided focused ultrasound for patients with treatment-refractory obsessivecompulsive disorder: a proof-of-concept study. Mol Psychiatry 20:1205-1211, 2015

19. Lindstrom PA: Prefrontal ultrasonic irradiation - a substitute for lobotomy. AMA Arch Neurol Psychiatry 72:399-425, 1954

20. Lipsman N, Schwartz ML, Huang Y, Lee L, Sankar T, Chapman M, et al: MR-guided focused ultrasound thalamotomy for essential tremor: a proof-of-concept study. Lancet Neurol 12:462-468, 2013

21. Meyers R, Fry WJ, Fry FJ, Dreyer LL, Schultz DF, Noyes RF: Early experiences with ultrasonic irradiation of the pallidofugal and nigral complexes in hyperkinetic and hypertonic disorders. J Neurosurg 16:32-54, 1959

22. Miller MW, Miller DL, Brayman AA: A review of in vitro bioeffects of inertial ultrasonic cavitation from a mechanistic perspective. Ultrasound Med Biol 22:1131-1154, 1996

23. Moser D, Zadicario E, Schiff G, Jeanmonod D: Measurement of targeting accuracy in focused ultrasound functional neurosurgery. Neurosurg Focus 32(1):E2, 2012

24. Nelson E, Lindstrom PA, Haymaker W: Pathological effects of ultrasound on the human brain. A study of 25 cases in which ultrasonic irradiation was used as a lobotomy procedure. J Neuropathol Exp Neurol 18:489-508, 1959

25. Ohl CD, Arora M, Ikink R, de Jong N, Versluis M, Delius M, et al: Sonoporation from jetting cavitation bubbles. Biophys J 91:4285-4295, 2006

26. Peters RD, Hinks RS, Henkelman RM: Heat-source orientation and geometry dependence in proton-resonance frequency shift magnetic resonance thermometry. Magn Reson Med 41:909-918, 1999

27. Pinton G, Aubry JF, Bossy E, Muller M, Pernot M, Tanter M: Attenuation, scattering, and absorption of ultrasound in the skull bone. Med Phys 39:299-307, 2012 
28. Sammartino F, Krishna V, King NK, Lozano AM, Schwartz ML, Huang Y, et al: Tractography-based ventral intermediate nucleus targeting: novel methodology and intraoperative validation. Mov Disord 31:1217-1225, 2016

29. Viallon M, Petrusca L, Auboiroux V, Goget T, Baboi L, Becker CD, et al: Experimental methods for improved spatial control of thermal lesions in magnetic resonance-guided focused ultrasound ablation. Ultrasound Med Biol 39:15801595,2013

30. Xu Z, Carlson C, Snell J, Eames M, Hananel A, Lopes MB et al: Intracranial inertial cavitation threshold and thermal ablation lesion creation using MRI-guided $220-\mathrm{kHz}$ focused ultrasound surgery: preclinical investigation. J Neurosurg 122:152-161, 2015

\section{Disclosures}

Mr. Blanke and Mr. Tilden are employees of Insightec Ltd. Dr.
Elias received clinical or research support for the study described (includes equipment or material) from Insightec Ltd.

\section{Author Contributions}

Conception and design: Elias. Drafting the article: all authors. Critically revising the article: Elias, Wang. Reviewed submitted version of manuscript: all authors. Approved the final version of the manuscript on behalf of all authors: Elias. Administrative/ technical/material support: Blanke, Tilden. Study supervision: Elias.

\section{Supplemental Information \\ Videos}

Video Abstract. https://vimeo.com/249835540.

\section{Correspondence}

W. Jeffrey Elias: University of Virginia, Charlottesville, VA. wje4r@hscmail.mcc.virginia.edu. 